\title{
Literature Review of the Extraction and Analysis of Trace Contaminants in Food
}

$$
\text { May } 21^{\text {st }}, 2010
$$

Task 1: Extraction and Identification of Chemical Signatures in Complex Matrices (Food) - U.S. Sweden Collaboration

Principal Investigator (PI): Audrey Martin Williams, Ph.D.

DHS Program Manager: Robert. L. Bull, Ph.D. and Robert Sibert, Ph.D.

\author{
Audrey Martin Williams, Armando Alcaraz \\ Forensic Science Center \\ Lawrence Livermore National Laboratory \\ 7000 East Ave. L-091 \\ Livermore, CA 94550
}

martin210@1ln1.gov, 925-423-4675

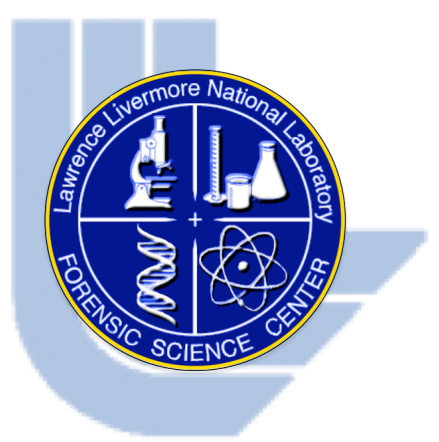

LLNL-TR-443692 


\section{Disclaimer}

This document was prepared as an account of work sponsored by an agency of the United States Government. Neither the United States Government nor the University of California nor any of their employees, makes any warranty, express or implied, or assumes any legal liability or responsibility for the accuracy, completeness, or usefulness of any information, apparatus, product, or process disclosed, or represents that its use would not infringe privately owned rights. Reference herein to any specific commercial product, process, or service by trade name, trademark, manufacturer, or otherwise, does not necessarily constitute or imply its endorsement, recommendation, or favoring by the United States Government or the University of California. The views and opinions of authors expressed herein do not necessarily state or reflect those of the United States Government or the University of California, and shall not be used for advertising or product endorsement purposes.

\section{Auspices Statement}

This work performed under the auspices of the U.S. Department of Energy by Lawrence Livermore National Laboratory under Contract DE-AC52-07NA27344. 


\section{Table of Contents}

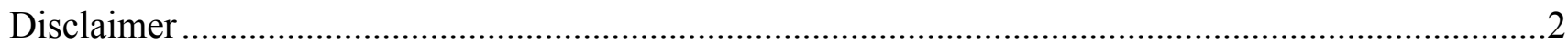

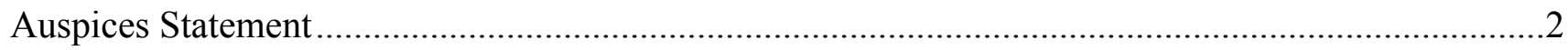

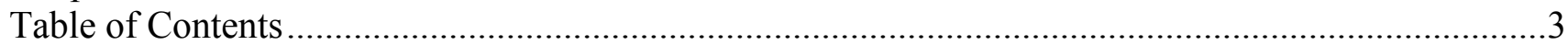

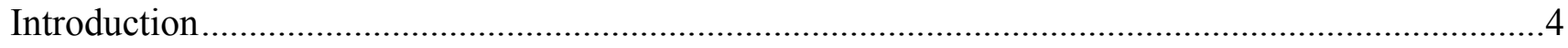

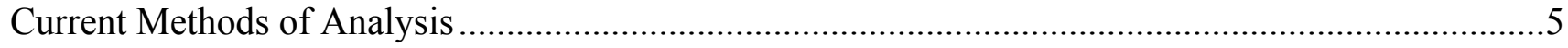

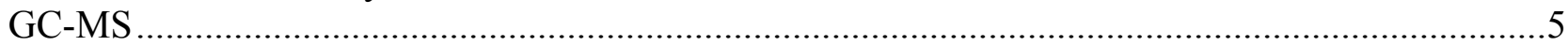

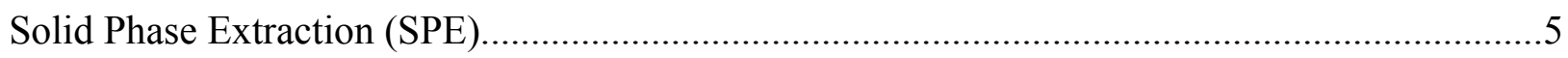

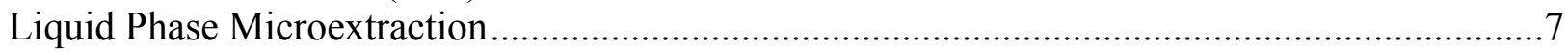

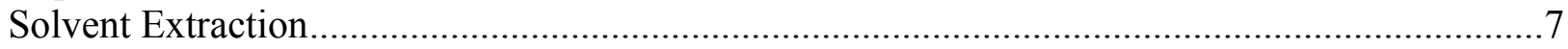

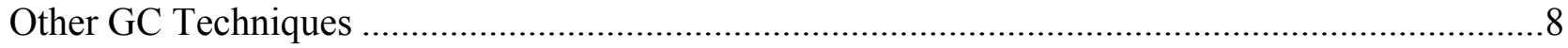

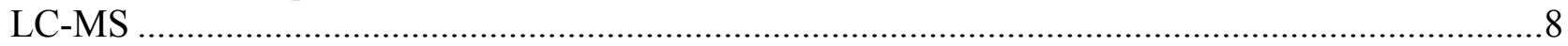

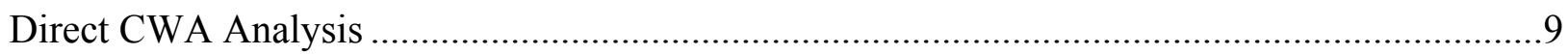

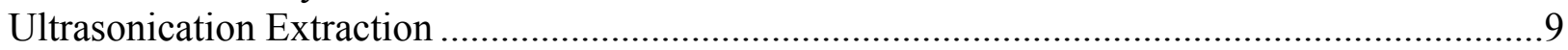

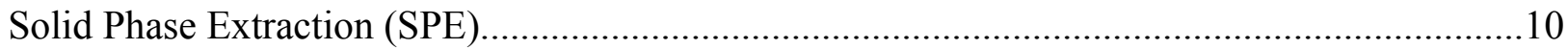

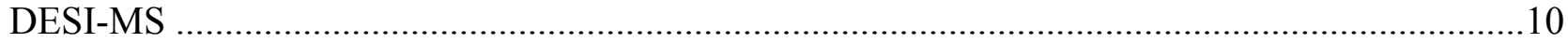

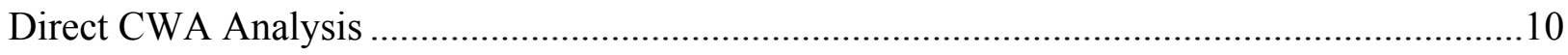

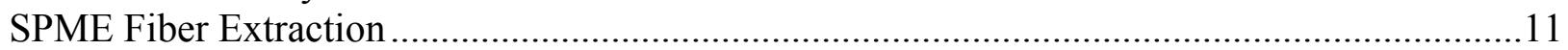

Nuclear Magnetic Resonance (NMR) .......................................................................... 12

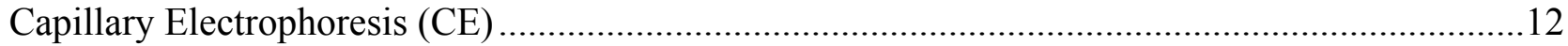

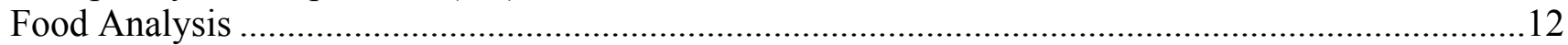

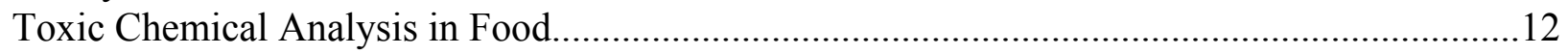

Analysis of CWA and CWA Degradation Products in Food.................................................13

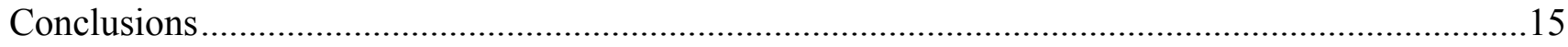

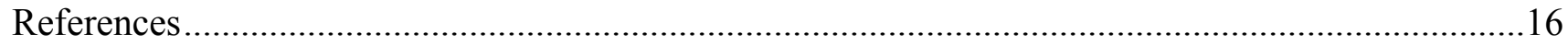




\section{Introduction}

There exists a serious concern that chemical warfare agents (CWA) may be used in a terrorist attack against military or civilian populations. While many precautions have been taken on the military front (e.g. protective clothing, gas masks), such precautions are not suited for the widespread application to civilian populations. Thus, defense of the civilian population, and applicable to the military population, has focused on prevention and early detection. Early detection relies on accurate and sensitive analytical methods to detect and identify CWA in a variety of matrices.

Once a CWA is detected, the analytical needs take on a forensic application - are there any chemical signatures present in the sample that could indicate its source? These signatures could include byproducts of the reaction, unreacted starting materials, degradation products, or impurities. Therefore, it is important that the analytical method used can accurately identify such signatures, as well as the CWA itself.

Contained herein is a review of the open literature describing the detection of CWA in various matrices and the detection of trace toxic chemicals in food. Several relevant reviews have been published in the literature, ${ }^{1-5}$ including a review of analytical separation techniques for CWA by Hooijschuur et al. ${ }^{1}$ The current review is not meant to reiterate the published manuscripts; is focused mainly on extraction procedures, as well as the detection of VX and its hydrolysis products, as it is closely related to Russian VX, which is not prevalent in the literature. It is divided by the detection technique used, as such; extraction techniques are included with each detection method. 


\section{Current Methods of Analysis}

Samples that contain chemical warfare agents are typically impure samples that contain multiple components, degradation products, and matrix materials. As such a complex mixture, separations are frequently necessary before the identification of the CWA contained therein.

\section{GC-MS}

Gas chromatography-mass spectrometry (GC-MS) is one of the main analytical methods used by the Organization for the Prohibition of Chemical Weapons (OPCW). The advantage of separation before mass spectrometric analysis has yielded a vast amount of data about CW signatures. GC-MS does rely on the compounds being analyzed to have some volatility in order for them to move through the GC column and reach the detector. Derivatization is often used to increase the volatility of an analyte, particularly hydrolysis products which are commonly less volatile than their parent agent.

\section{Solid Phase Extraction (SPE)}

Subramaniam et al. used GC-MS to evaluate several extraction methods for alkylphosphonic acids. ${ }^{6}$ SPE using high performance anion exchange extraction disks was used. An aqueous sample was introduced to the disk, followed by an acetonitrile wash and drying. N,O-bis-trimethylsilyltrifluoroacetamide (BSTFA), a common derivatizing agent, was then added to the vial and heated, followed by injection into the GC-MS. A DB-5MS column was used for separation, with helium as the carrier gas. The LODs were reported to be $0.14 \mathrm{ppb}$ when operated in SIM mode.

Kataoka et al. used macroporous strong anion-exchange (MSA) and strong cation-exchange (SCX) for the GC-MS analysis of methylphosphonic acid and alkyl methylphosphonic acids after 
derivatization with N-methyl-N-(tert-butyldimethylsilyl)trifluoroacetamide (MTBSTFA). ${ }^{7}$ Soil samples were vortexed and sonicated in water, centrifuged, and the supernatant filtered through a cellulose membrane, then subjected to pretreatment using SCX or MSA, derivatized, and analyzed via GC-MS. Water samples, as well as cola and coffee drinks, were filtered, pretreated, derivatized, and analyzed in the same manner. Pretreatment using MSA was determined to be the most efficient method of eliminating interferents in the derivatization process for all compounds. Detection limits in the soil samples were reported as $0.12-0.18 \mu \mathrm{g} / \mathrm{g}$ of soil for all compounds.

Saradhi et al. used ion pair SPE (IP-SPE) to extract alkylphosphonic acids from aqueous samples. The aqueous sample containing the compounds of interest was mixed with a derivatizing solution (phenyltrimethyl ammonium hydroxide) and passed through an activated charcoal, C18, DSC-6S, or Oasis HLB SPE cartridge, washed with methanol, and the extract concentrated and injected into the GC. ${ }^{8}$ Optimal recovery ( $98 \%$ ) was achieved with the activated charcoal cartridges; the polymeric cartridges (DSC-6S and HLB) showed poor recoveries.

Kanaujia et al. compared the use of strong anion-exchange AccuBond II (SAX) SPE cartridges to Oasis mixed mode anion exchange (MAX) cartridges. ${ }^{9}$ Water samples were passed through the cartridges, dried, and the alkylphosphonic acids were eluted with acidic methanol. After drying, the sample was derivatized using BSTFA and injected into the GC-MS. MAX cartridges were found to perform better, with $30-40 \%$ higher extraction efficiencies. Limits of detection as low as $0.5 \mathrm{ng} / \mathrm{mL}$ were reported. 


\section{Liquid Phase Microextraction}

Pardasani et al. has developed a liquid phase microextraction (LPME) method that combines derivatization, extraction, and concentration into a single step. ${ }^{10}$ A contaminated water sample was basified with potassium carbonate, and propyl bromide was added for derivatization and heated for 120 min. Sodium chloride was then added to the sample, and a solvent (trichloroethylene) filled hollow fiber was inserted in to the sample. After 30 minutes, the solvent from the fiber was removed and injected in the GC. Limits of detection of $0.5-0.75 \mu \mathrm{g} / \mathrm{mL}$ and $0.1-0.5 \mu \mathrm{g} / \mathrm{mL}$ were reported for dibasic and monobasic alkylphosphonic acids, respectively.

\section{Solvent Extraction}

D'Agostino et al. used GC-MS to analysis soil samples from a mustard storage site. The soil was extracted in dichloromethane, centrifuged, and the supernatant injected into the GC. Although mustard itself was not detected, several hydrolysis products, including thiodiglycol, were detected. Some of these products, including 1,4-oxathiane and 14-dithiane, could not be detected in parallel analyses of an aqueous extract using LC-ESI-MS. ${ }^{11}$

Bonierbale et al. developed a method for the analysis of VX and diisopropylaminoethanethiol (DPAT, a degradation product of VX) in rat plasma using GC-MS and GC-NPD. ${ }^{12}$ Samples were extracted using 50:50 ethyl acetate:hexane. A thiol reducing agent was added for the detection of DPAT. The organic phase was then injected into the GC. Extraction yield were $87 \pm 10$ and $92 \pm 15 \%$ for VX and DPAT, respectively, and limits of detection were 0.2 or $0.3 \mu \mathrm{M}$. 


\section{Other GC Techniques}

Degenhardt-Langlaan et al. have developed a GC-thermionic detection (TID)/flame ionization detection (FID) method for the analysis of a mixture of sarin, soman, tabun, VX, and diisopropyl fluorophosphates (an insecticide) in water. ${ }^{13}$ SPE using an Amberlite XAD-4 column followed by extraction with n-pentane/methanol $(95: 5 \mathrm{v} / \mathrm{v})$ was used to isolate the sample. The target compounds were detected at the ppt level in a $10 \mathrm{~mL}$ sample.

Sega et al. used GC-flame photometric detection (FPD) to analyzed alkylphosphonic acids in ground water. ${ }^{14}$ BakerBond Quaternary Amine and Aminopropyl SPE columns were used to clean up the samples. A vacuum manifold was attached to the SPE column, which would draw the water sample $(50 \mathrm{~mL})$ through the column, which was then rinsed with methanol. Compounds were eluted from the column using trimethylphenyl ammonium hydroxide (TMPAH) which derivatized the phosphonic acids to their methyl esters in the injection port of the GC. Concentrations as low as $125 \mu \mathrm{g} / \mathrm{L}$ were detected.

\section{LC-MS}

LC-MS is also commonly used by OPCW for the analysis of CWA. LC-MS is often chosen over GC-MS, as the CWA and many of their hydrolysis and degradation products can be analyzed without the need for derivatization that is necessary in GC-MS. ${ }^{11}$

Several ionization methods coupled to LC-MS have been reported for the detection of CWA and their degradation products. ${ }^{15}$ Electrospray ionization (ESI) is most commonly used and utilizes packed 
capillary separation on column, which results in lower flow rates. Gradient mobile phases are commonly employed to analyze compounds with a range of size and polarity. ${ }^{15}$

\section{Direct CWA Analysis}

D'Agostino et al. at Defence Research and Development Canada have analyzed many CWA using LC-ESI-MS. ${ }^{15}$ Matrices studied have included water, soil, snow, and munitions. Separation was performed using a water (1\% TFA):acetonitrile gradient mobile phase on a C18 column. While mustard did create problems as it did not ionize easily using ESI, the other CWA and hydrolysis products gave acceptable results. Specifically, they studied the degradation products of VX via LCESI-MS. ${ }^{16}$ A 15 year old VX sample, shown to contain only $10 \% \mathrm{VX}$ in the volatile organic content, was analyzed and 38 components were detected, 26 of which were identified. ${ }^{16}$ VX itself was not detected; however, it was hypothesized that it hydrolyzed prior to, or during, analysis.

Wils and Hulst used thermospray-LC-MS to detect several alkylphosphonic acids. ${ }^{17}$ Ammonium acetate and several tetraalkylammonium salts were tested as ion-pair reagents. Detection limits of $100 \mathrm{pg}$ of dimethylthiophosphoric acid using ammonium acetate as the ion-pair reagent were reported.

\section{Ultrasonication Extraction}

D'Agostino et al. also studied the extraction of CWA from office media including paper, drywall, carpet, and office fabric. ${ }^{18}$ The CWA were extracted from the media using water and ultrasonic extraction. The extract was then centrifuged and analyzed via LC-ESI-MS. Water was chosen for 
the extraction as it contained less co-extracted interferences than organic extracts, and was less destructive to the media. Reported recoveries were between $20-100 \%{ }^{18}$

\section{Solid Phase Extraction (SPE)}

Wils and Hulst tested solid phase extraction for the preconcentration of pinacolylmethylphosphonic acid. ${ }^{17}$ Sep-Pak C18 cartridges were equilibrated in tetrabutylammonium hydroxide, to which an aqueous solution containing the compound of interest and tetrabutylammonium hydroxide was added. Methanol was then used to elute the ion pair, which was evaporated down to $200 \mu \mathrm{L}$ and analyzed via LC-MS. A preconcentration factor $>100$ was achieved, and the recovery was described around $90 \%$. Wils and Hulst also studied the analysis of VX in water and soil samples using thermospray-LCMS. ${ }^{19}$ In order to avoid co-elution of VX with a common plasticizer, a mobile phase of acetonitrile:methanol:ammonium acetate (70:20:10) was used. Directly injected water allowed a detection limit of $5 \mathrm{ng} / \mathrm{mL}$ for VX. Use of a Sep-Pak C18 or Bond Elut C18 SPE cartridge preconcentrated the VX. In fact, a water sample could be stored on a Bond Elut C18 cartridge for 18 hr without decomposition, which would allow for field collection directly onto the SPME cartridge. Using SPE, the limit of detection for VX was reported to be $0.1 \mathrm{mg} / \mathrm{mL} .^{19}$

\section{DESI-MS}

Direct CWA Analysis

D'Agostino et al used desorption electrospray ionization (DESI)-MS to demonstrate the detection of several CWA and hydrolysis products using a 'dip and shoot' method. ${ }^{20}$ A fused silica or stainless steel probe was dipped into a solution of each compound, and the tip held in the spray of the DESI interface. Successful detection of GB, GD, GF, GA, and VX was reported. The dip and shoot 
method was also demonstrated on solutions of hydrolysis products of each agent, including ethyl methylphosphonic acid (VX hydrolysis product), isopropyl methylphosphonic acid (GB hydrolysis product), pinacolyl methylphosphonic acid (GD hydrolysis product), and thiodiglycol (mustard hydrolysis product).

Song and Cooks used reactive DESI-MS/MS to detect CWA hydrolysis products deposited on Teflon surfaces, as well as spiked human urine on a glass slide. ${ }^{21}$ Boric acid $(0.5 \%$ in a 1:1 methanol:water solution) was used as the spray reagent, which creates borate anions that react with the compounds of interest. Methylphosphonic acid, ethyl methylphosphonic acid, and isopropyl methylphosphonic acid were all detected, with LODs of $<0.1 \mathrm{ng}$ in the urine sample.

\section{SPME Fiber Extraction}

D'Agostino et al. used SPME fibers to preconcentrate the headspace above office media contaminated with CWA. ${ }^{18}$ A PDMS/DVB (65 $\mu \mathrm{m}$ film thickness) SPME fiber was placed in the headspace above a carpet sample that had been spiked with GB, GD, or triethyl phosphate (TEP) for 3 minutes at room temperature. The SPME fiber was then placed between the electrospray needle and the sampling cone of a DESI system. GB, GD, and TEP were successfully identified in this manner. D'Agostino et al. also used ultrasonication with water to extract mustard and thiodiglycol from sand samples. After extraction, a PDMS/DVB SPME fiber was dipped in the extract (dip and shoot) and analyzed via DESI-MS. ${ }^{20}$ A 50:50 acetonitrile:water (0.1\% TFA) was used as the spray solvent. Only thiodiglycol was detected in the sample, as the mustard was completely hydrolyzed. Hydrolysis products of several of the agents studied could also be successfully sampled in this manner. ${ }^{22}$ 


\section{Nuclear Magnetic Resonance (NMR)}

NMR is commonly used in identification of chemical warfare agents. The heteronucleii, ${ }^{19} \mathrm{~F}$ and ${ }^{31} \mathrm{P}$, present in many CWAs provide splitting patterns and coupling constants that can aid in compound identification. The application of NMR to the identification of CWA in complex matrices has been relatively limited. Although ${ }^{31} \mathrm{P}$ NMR can be used to identify some $\mathrm{CW}$ in complex mixtures, confirmation is usually required using an additional technique.

Mazumder et al. developed an LC-UV-NMR method to identify alkyl alkylphosphonic acids and alkylphosphonic acids and applied it to a water sample. ${ }^{23}$ Oasis MAX SPE cartridges were used to extract the analytes, which were eluted in acidic methanol, re-dissolved in acetonitrile after evaporation, and reacted with phenyl diazomethane to benzylate the analytes. Limits of detection of 16-30 $\mu \mathrm{g}$ were obtained for all analytes.

\section{Capillary Electrophoresis (CE)}

Kataoka et al. also analyzed methylphosphonic acid and alkyl methylphosphonic acids using CE with indirect UV absorption at $254 \mathrm{~nm}$. Detection limits of approximately 2.5 ppm were achieved. ${ }^{7}$

\section{Food Analysis}

Toxic Chemical Analysis in Food

Saito et al. reported using GC-MS and LC-MS/MS to detect several hundred rodenticides and pesticides in bovine milk. ${ }^{24}$ Compounds were extracted in acetonitrile and salted out using sodium chloride. In some cases, an additional step of n-hexane partitioning and PSA cartridge column 
chromatograph was used. The relative standard deviations of the recovery of each drug were within $28 \%$.

Nishina et al. reported a GC-MS method that detected 340 pesticides spiked into Chinese cabbage at 0.05 ppm, and an LC-MS method that detected 220 pesticides in the same matrix. ${ }^{25}$ Two different extraction methods were used: supercritical fluid extraction (SFE) and acetonitrile extraction.

Sun et al. described the use of LC-MS-MS for the detection of melamine in milk samples. ${ }^{26}$ Cleanert PCX-SPE cartridges were used for sample extraction. The liquid sample was treated with trichloroacetic acid and lead acetate to eliminate proteins and extract the analyte. The solution was then centrifuged, and the supernatant introduced to the SPE cartridge. Melamine was eluted using 1:20 ammonia:methanol, which was evaporated to dryness, and redissolved in 1:4 methanol:water. The solution was filtered through a mixed cellulose ester membrane and introduced to the LC column. $91-100 \%$ recovery was reported for raw milk samples, and the LOD of the method was $18 \mu \mathrm{g} / \mathrm{kg}$.

\section{Analysis of CWA and CWA Degradation Products in Food}

Several documents of note described the analysis of food or drink matrices for CWA or CWA degradation products. D'Agostino et al. analyzed bottled water, canola oil, and corn meal for contamination by GA, GB, GD, GF, VX, mustard and TEP using DESI-MS and LC-ESI-MS (only bottled water sample). ${ }^{22}$ For SPME analysis, a PDMS/DVB fiber was inserted into an aliquot of the bottled water for 2 minutes, canola oil (heated to 40-80C) for 10 minutes, and corn meal (heated to 40-80C) for 10 minutes. The SPME fiber was then placed in the path of the DESI spray. By using 
DESI-MS/MS GD and TEP could not be distinguished due to the same nominal mass of the $[\mathrm{M}+\mathrm{H}]+$ ion. GD and GF showed very low signals when sampled out of the headspace of the canola oil or corn meal. VX and H could not be detected in the canola oil or corn meal samples.

Kolakowski et al. studied CWA and alkylphosphonic acids in food products using high field asymmetric waveform ion mobility spectrometry atmospheric pressure ionization mass spectrometry (FAIMS-APCI-MS), FAIMS-ESI-MS and LC-ESI-MS. ${ }^{27}$ GA, GB, GD, GF, and several alkylphosphonic acids were spiked into bottle water, canola oil, and cornmeal or honey. Bottled water was diluted in 75:25 acetonitrile:water and directly injected. Canola oil, cornmeal, or honey was diluted in 75:25 acetonitrile:water, vortexed, and the upper layer passed through a syringe filter prior to injection. FAIMS-ESI-MS and FAIMS-APCI-MS were unable to separate the four CWA studied; however, FAIMS does present a lower background when the spiked samples were studied. The limits of detection for the CWA in standard solutions were $0.5-1.4 \mathrm{ng} / \mathrm{mL}$, and $1-35 \mathrm{ng} / \mathrm{mL}$ for the spiked foods. Several of the degradation products could not e determined in the complex matrices studied. This was hypothesized to $b$ e due to matrix interference, or poor ionization efficiency.

Kubachka et al. described the detection of CWA degradation products in food samples using LC-ICPMS with ${ }^{31} \mathrm{P}$-specific detection and LC-ESI-MS. ${ }^{28}$ Several separation characteristics were used in order to separate many alkylphosphonic acids, as well as phosphoric acid. Liquid samples, bottled water, grape juice, apple juice, soda, and sports drink, were diluted 1:10 or 1:20 with doubledeionized water and injected into the LC. More complex food samples, lettuce and infant formula, were aqueously extracted before analysis. 
Owens and Koester analyzed beverages using LC-MS-MS. ${ }^{29,30}$ Apple juice, bottled water, cola, whole milk, and juice drink were spiked with several alkylphosphonic acids. ${ }^{29}$ Samples were extracted using two Strata-X SPE cartridges in series after acidification with formic acid. Phosphonic acids were eluted using a gradient of water/formic acid, 50:50 methanol:acetonitrile, and methanol/formic acid, and the eluent injected into the LC. Recoveries ranged from 38.1-110.4\%, with the exception of methylphosphonic acid, which had recoveries of $15.8-33.2 \%$. This was hypothesized to be due to the low molecular weight of the compound, it's early elution time, and

matrix interference. Several beverages were also spiked with abrine. ${ }^{30}$ Samples were extracted using liquid-liquid extraction or Strata-X SPE cartridges and the analyte eluted with methanol. Recoveries of $88-111 \%$ were reported, with a limit of detection of $0.025 \mu \mathrm{g} / \mathrm{mL}$. SPE cartridges were found to have increased recovery versus liquid-liquid extraction.

\section{Conclusions}

Several detection strategies have been applied to the identification of CWA and CWA degradation products in various matrices, both simple, and complex. Chromatographic methods provide the separation step that is necessary in simultaneously screening samples for a variety of toxic components. GC-MS is useful in CWA identification; however, derivatization is often necessary to detect and identify CWA degradation products that may not have the appropriate volatility for GCMS analysis. LC-MS is also useful in CWA identification, and avoids the derivatization step that is necessary for GC-MS.

Several extract techniques have also been used for CWA and CWA degradation product analysis. SPE is a particularly appealing technique, in that the cartridge can be selected for the analytes of 
interest, and the eluting solvent can be modified. Recoveries tend to be quite high using SPE, resulting in accurate detection capabilities.

\section{References}

(1) Hooijschuur, E. W. J.; Kienz, C. E.; Brinkman, U. A. T. Journal of Chromatography A 2002, $982,177-200$.

(2) Papouskova, B.; Bednar, P.; Bartak, P.; Frycak, P.; Sevcik, J.; Stransky, Z.; Lemr, K. J. Sep. Sci. 2006, 29, 1531-1538.

(3) Kientz, C. E. Journal of Chromatography A 1998, 814, 1-23.

(4) Black, R. M.; Muir, B. Journal of Chromatography A 2003, 1000, 253-281.

(5) Kanamori-Kataoka, M.; Seto, Y. J. Health Sci. 2008, 54, 513-523.

(6) Subramaniam, R.; ₹stot, C.; Nilsson, C.; $\div$ stin, A. Journal of Chromatography A 2009, 1216, $8452-8459$.

(7) Kataoka, M.; Tsuge, K.; Seto, Y. Journal of Chromatography A 2000, 891, 295-304.

(8) Vijaya Saradhi, U. V. R.; Prabhakar, S.; Jagadeshwar Reddy, T.; Murty, M. R. V. S. Journal of Chromatography A 2007, 1157, 391-398.

(9) Kanaujia, P. K.; Pardasani, D.; Gupta, A. K.; Kumar, R.; Srivastava, R. K.; Dubey, D. K. Journal of Chromatography A 2007, 1161, 98-104.

(10) Pardasani, D.; Kanaujia, P. K.; Gupta, A. K.; Tak, V.; Shrivastava, R. K.; Dubey, D. K. Journal of Chromatography A 2007, 1141, 151-157.

(11) D’Agostino, P. A.; Hancock, J. R.; Chenier, C. L.; Lepage, C. R. J. Journal of Mass Spectrometry 2003, 9, 609-618. 
(12) Bonierbale, E.; Debordes, L.; Coppet, L. Journal of Chromatography B: Biomedical Sciences and Applications 1997, 688, 255-264.

(13) Degenhardt-Langelaan, C. E. A. M.; Kientz, C. E. Journal of Chromatography A 1996, 723, 210-214.

(14) Sega, G. A.; Tomkins, B. A.; Griest, W. H. Journal of Chromatography A 1997, 790, $143-$ 152.

(15) D’Agostino, P. A.; Chenier, C. L.; DRDC Suffield: Alberta, CAN, 2006.

(16) D’Agostino, P. A.; Hancock, J. R.; Provost, L. R. Journal of Chromatography A 1999, 837, 93-105.

(17) Wils, E. R. J.; Hulst, A. G. Journal of Chromatography A 1988, 454, 261-272.

(18) D'Agostino, P. A.; Hancock, J. R.; Chenier, C. L.; Lepage, C. R. J. Journal of Chromatography A 2006, 1110, 86-94.

(19) Wils, E. R. J.; Hulst, A. G. Journal of Chromatography A 1990, 523, 151-161.

(20) D’Agostino, P. A.; Chenier, C. L.; DRDC Suffield: Alberta, CAN, 2009.

(21) Song, Y.; Cooks, R. G. Journal of Mass Spectrometry 2007, 42, 1086-1092.

(22) D’Agostino, P. A.; Chenier, C. L.; DRDC Suffield: Alberta, CAN, 2007.

(23) Mazumder, A.; Gupta, H. K.; Garg, P.; Jain, R.; Dubey, D. K. Journal of Chromatography A 2009, 1216, 5228-5234.

(24) Saito, M.; Kozutsumi, D.; Kawasaki, M.; Kanbashi, M. Shokuhin Eiseigaku Zasshi 2008, 49, $228-238$.

(25) Nishina, T.; Murakawa, H.; Fukushima, K.; Tobino, T. Kumamoto-ken Hoken Kankyo Kagaku Kenkyushoho 2006, 35, 7-85.

(26) Sun, H.; Wang, L.; Ai, L.; Liang, S.; Wu, H. Food Control, 21, 686-691. 
(27) Kolakowski, B. M.; D’Agostino, P. A.; Chenier, C.; Mester, Z. n. Analytical Chemistry 2007, $79,8257-8265$.

(28) Kubachka, K. M.; Richardson, D. D.; Heitkemper, D. T.; Caruso, J. A. Journal of Chromatography A 2008, 1202, 124-131.

(29) Owens, J.; Koester, C. Journal of Agricultural and Food Chemistry 2009, 57, 8227-8235.

(30) Owens, J.; Koester, C. Journal of Agricultural and Food Chemistry 2008, 56, 11139-11143. 